\title{
PHARMACEUTICAL SEARCH ENGINE
}

\author{
Ghadeer Aldweik, Saadia Malik, Abrar Almuhammidi, Wejdan Alyoubi, \\ Ahad Alsulami and Hind Al-Oufi \\ Faculty of Computing and Information Technology in Rabigh, King Abdulaziz University, Saudi Arabia
}

\begin{abstract}
The vertical search engine searches in the text of specific domain. In this project, we built a pharmaceutical vertical search engine using a supervised learning classifier, Rocchio, to classify documents into two different classes; one pharmaceutical and another computer science. For learning of the classifier, small document collection is created. It is evaluated using abstracts from 86 research papers and accuracy yields $90 \%$ results. An inverted index is built containing terms from selected pharmaceutical documents. An interface is also developed to interact with the user. User can issue simple keyword like queries and documents are retrieved using TF-IDF statistics and BM25 weighting scheme. Retrieved results are ranked in descending order from the highest relevance score to lowest relevance score. New information can be classified and added to the index using search interface. The system is designed and developed using the Spiral Model and implemented in dot.net tools. The survey and interviewing techniques are also used to identify the needs and prioritizing tasks.
\end{abstract}

\section{KEYWORDS}

Vertical Search Engine, Pharmaceutical, Classifier, BM25 Weighting

\section{INTRODUCTION}

With the exponential growth of the World Wide Web content, it is becoming more and more difficult to find out what we want. A general-purpose search engine such as Google, Yahoo and Bing provides a long list of retrieved results with high coverage and sometimes low precision. A user spends a lot of time and effort in finding the relevant information.

A general-purpose search engine consists of four components. It includes text acquisition, indexing, query processing, and a search interface. The purpose of text acquisition is to identify and acquire the documents. This can be an existing collection or documents/web pages can be crawled from the internet. Crawler can be of different types (Croft et al., 2015); general-purpose, focused or vertical and enterprise. The general-purpose crawler traverses all the contents from the web graph. While focused or vertical crawler use classification techniques to restrict traversing to a specific domain. The specific domain can be based on topicality, media type or genre of the document. This type of crawler is used by vertical or topical search engines such as providing access to medical information only. During the indexing, documents are transformed, and an inverted index is created. The query processing component encompasses a searcher interface to interact with the user, a ranking scheme to rank the retrieved documents from the index in descending order of relevance.

There are certain domains where precision is more critical than the other such as Medical \& pharmaceutical. In a recent article published (Anon, 2019), 60\% of online search results for pharmaceutical drug leads to fakes.

There are several potential benefits of vertical search engines over general-purpose search engines. Firstly, greater precision can be achieved due to the limited scope. Secondly, unique user tasks with fewer efforts to prune the results can be supported.

This paper is organized as follows. Section 2 discusses the related work. Section 3 describes the methodology details; Section 4 presents the evaluation results and Finally, conclusion and an outlook to future work is presented in Section 5. 


\section{RELATED WORK}

A focused crawler is topic specific and its purpose is to gather particular web pages from the internet that are pertinent to a given subject. There are two most important tasks for the crawler; selecting the web pages and classifying them into topics (Harrell, 2015). For selecting the webpage, link-based weighting methods are used (Chakrabarti et al., 2002, Davison, 2000). The other task of the crawler is to classify the relevant pages from the irrelevant one depending upon the given topic.

Text classification (Kowsari et. al, 2019). is one of the significant research issues in the field of text mining, where the documents are classified with supervised knowledge. Supervised classifiers are group of machine learning and data mining techniques that attempt to assign the document a label from set of pre-known labels.

The most common approach used in text classification is generally known as Term Frequency Inverse Document Frequency (TF-IDF) (Jones, 1972, Jones, 2004). Traditional techniques include Rocchio classification. Details are presented in Methodology section. Logistic regression (LR) has been applied in many different data mining domains (Harrell, 2015). The Naïve Bayes Classifier (NBC) was very popular in the earlier history of information retrieval (Larson, 2010).

Other discriminatory and -parametric techniques have been studied and used as classification tasks such as k-nearest neighbor (KNN (Li et al., 2001), Support Vector Machine (SVM) (Manevitz et al., 2001) and Tree-based classifiers such as decision tree and random forest (Xu et al., 2012). Detailed survey of text classification algorithm is presented in (Kowsari et. al, 2019).

Another important aspect of the search engine is to return the relevant documents upon its similarity to query given by the user. There are a variety of methods and approaches to compute the similarity (Croft, 2015, Manning, 2008, Baeza-Yates \& Ribeiro-Neto, 2011). Some are based on basic criteria such as Boolean match while the other uses advanced approaches such as hyperlink structure of web graph such as PageRank (Brin et. al, 1998), HITS (Kleinberg, 1999.), SALSA (Lempel et al., 2001), anchor text information (Craswell et al., 2001, Fujii, 2008) and user-click behavior (Baeza-Yates et al., 2006).

The focused search engines can be based on topicality, media type or genre of document. Medical Information Retrieval is an example of focused retrieval. It refers to the technologies and techniques used to search and access and organize the medical information (Goeuriot et al., 2016). Graph inference retrieval model to improve the biomedical IR is presented in (Koopman et al., 2016). A novel approach for the extraction biomedical terminology is proposed (Lossio-Ventura et al., 2016). Semantic search engines incorporate not only the keywords but also on their semantic(meaning) intelligently based on Semantic Web Technologies (Tümer, et al., 2009, Madhu et al., 2011).

\section{METHODOLOGY}

Spiral model is a systems development method (SDM) used in information technology. This model is a combination of iterative development process model and sequential linear development model. This model is used to construct the system Pharmaceutical Search Engine (PSE).

The spiral model has four phases. A software project repeatedly passes through these phases in iterations called Spirals. The phases of spiral model are Identification, Design, Construct or Build and finally Evaluation and Risk Analysis. A software project repeatedly passes through these phases in iterations (called Spirals).

For the identification phase, questionnaire and interviewing approach has been used. The Questionnaire is comprised of 7 questions primarily focusing on evaluating the need of this project. Two pharmacists are also interviewed to prioritize the requirements. 


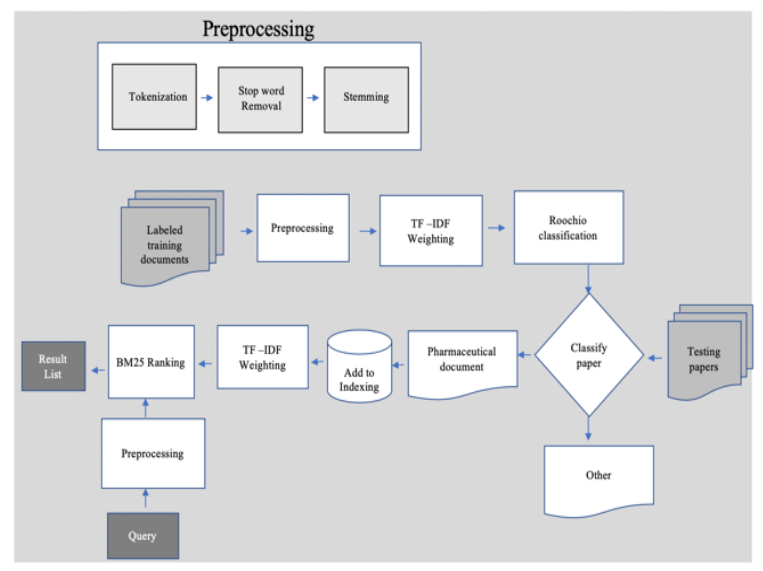

Figure 1. PSE Framework

The survey is conducted using Google Questionnaire. A total of 30 participants filled in the survey. The survey results showed that above $70 \%$ of the respondents indicated the difficulty in finding the required information using general search engines, around $80 \%$ of the respondents would prefer using the vertical pharmaceutical search engine. This study supports the idea of development of pharmaceutical search engine.

For the design, the requirements for the PSE system are determined through functional, non-functional and interface requirements. Two main actors are identified; one administrator and another user.

The functional requirements of administrate are as follows:

1. She/he can add/view papers information to database

2. She/he can insert/delete stop word to database

3. She/he can reduce words into their "roots" (base) and

4. She/he can delete/edit papers information from database by title or subject

5. Classify new document and add it to the database.

The functional requirements of the user are

1. Search about Pharmaceutical Information

2. Browse Pharmaceutical information that is provided by system

For the construction phase, tools used are C\#, asp, Visual Studio 2010, SQL Server. JScript and CSS (Cascading Style Sheet).

The technique developed for the PSE comprises of a few steps. The initial step is the collection and preparation of the data for supervised learning of the classifier. The pre- processing step incorporates tokenization, stop word removal and stemming. At that point the estimation of each term or weighting is given to the terms using TF-IDF weighting scheme. The consequent stage is the classification modeling with Rocchio algorithm to classify the testing data. After classification, the new document is added to the index. Whenever user gives a query, it is searched in the indexed documents and ranked list of results is prepared based on BM25 weighting scheme. The Figure 1 shows the complete framework of PSE. All the stages are discussed in detail below:

\subsection{Data Preparation}

Administrator can add the training paper to the database. The training set of the papers is a set of documents, each labeled with class. The screen is like, as shown in figure 2, with the exception that class code is not mentioned here. The details of the training and testing set is given in Evaluation section. The following information is required for each paper:

Paper no: It is paper number and it is unique

Paper title: It is the title of a paper.

Paper class code: It identifies document class. There are two classes; 1 for pharmacology and 2 for Computer Science. 
Paper subject: It determines the subject of the paper such as operating system, Medical pharmacology, pharmacology.

Paper abstract: For training, the whole contents of the documents are used while for testing only abstract of the paper is used for the classification purpose.

Administrator can browse/edit/delete the inserted papers as shown in figure 3.

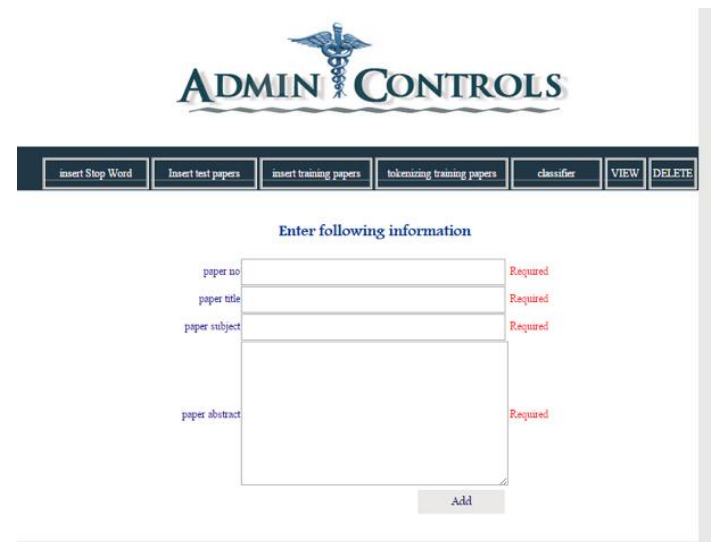

Figure 2. Adding testing paper

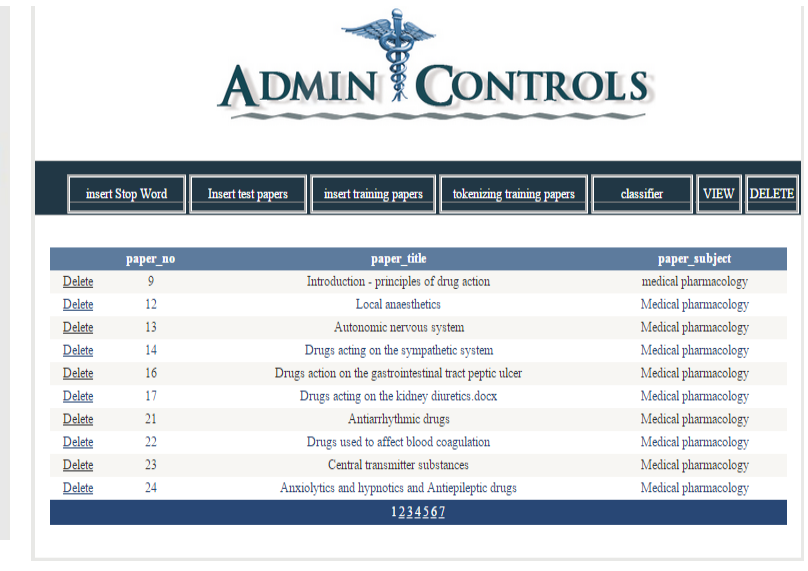

Figure 3. Browsing the pharmaceutical papers in the database

\subsection{Preprocessing}

The preprocessing step is applied at two different stages of the search engine. Firstly, when document is processed for the classification. Secondly, when user issues the query It consists of several step: tokenization, stop word removal, and finally the stemming process.

Tokenization is a process of breaking up a sentence into pieces such as words, phrases, keywords. The punctuation marks, symbols and numbers are discarded during this process.

Stop word removal is a process of removing irrelevant and meaningless terms from the document. Each document word is compared with the stop word list. The existing stop-word list is shown in Figure 4.

Stemming is the process of converting the words to roots from their derived forms. It suggests crude affix chopping language dependent e.g. automate(s), automatic, automation all reduced to automat. The most common algorithm, Porter's algorithm (Porter, 1980), for stemming English is used. It has repeatedly been shown to be empirically very effective.

\subsection{TF-IDF Weighting}

After the preprocessing, weights are assigned to each term. The well-known method for assigning weights is TF-IDF (Term Frequency Inverse Document Frequency) (Jones, 1972). It calculates Term Frequency (TF) and Inverse Document Frequency (IDF) values on each token (word) from documents. These assigned weights are used in classification and retrieval tasks. TF-IDF is the weight value of a word taken from the TF value and the inverse DF value, which is defined by Eq. I and 2.

TF: The frequency(f) of term $(t)$ in document(d) is defined as the number of times that $t$ occurs in $d$.

DF: the document frequency of $t$ is the number of documents that contain $t$

Paper length: It is the number of words of training documents

IDF: Inverse Document Frequency of $t$ by the following equation ( $N=$ number of documents)

TF-IDF Weight: The TFIDF weight of a term is the product of its TF weight and its IDF weight.

$$
\begin{gathered}
\text { idf }_{t}=\log _{10}\left(N / \mathrm{df}_{t}\right) \\
\mathrm{W}_{t, d}=\left(1+\log \mathrm{tf}_{t, d}\right) \times \log _{10}\left(N / \mathrm{df}_{t}\right)
\end{gathered}
$$




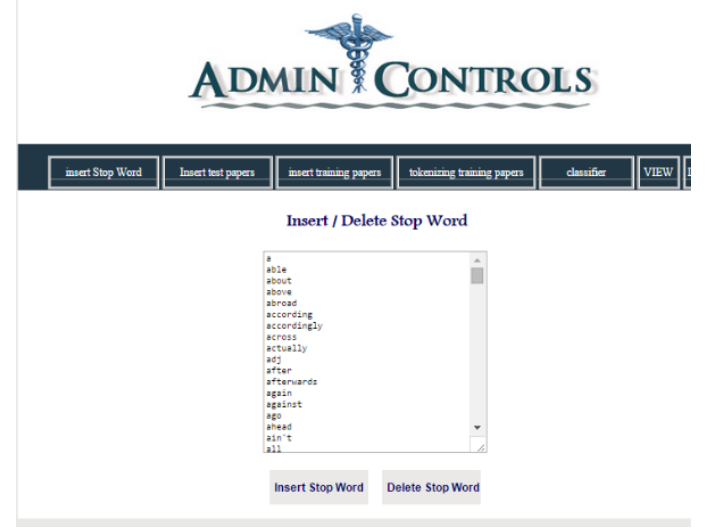

Figure 4. Insert/Delete Stop Words

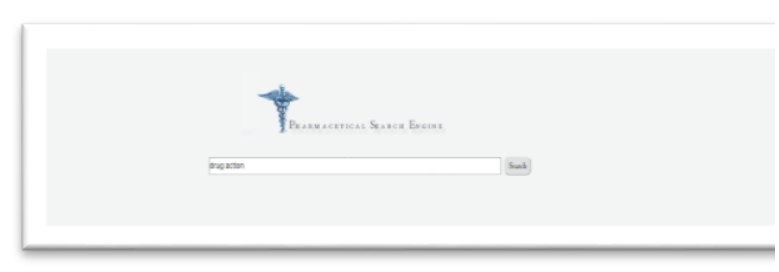

Figure 5. User issues the query

\subsection{Rocchio Classification}

Rocchio algorithm (Baeza-Yates \& Ribeiro-Neto, 2011) was first presented as relevance feedback method. Later it was adapted for the text and document classification task (Kowsari et. al, 2019). It considers TF-IDF weights for the terms instead of the Boolean features. Using the training documents, centroid vector or prototype vector is computed for each class. The centroid vector (in Eq. 3) of a class is the vector average or the center of the mass of its members. Normalized centroid is obtained by Eq. 4

$$
\vec{\mu}(c)=\frac{1}{\left|D_{c}\right|} \sum_{d \in D_{c}} \vec{v}_{d}
$$

where Dc is the set of documents in D that belongs to class $\mathrm{c}$ and $\overrightarrow{\mathrm{v}_{\mathrm{d}}}$ is the weighted vector representation of document $d$.

$$
\vec{\mu}_{c}=\frac{\sum_{d \in D_{c}} \vec{v}_{d}}{\left\|\sum_{d \in D_{c}} \vec{v}_{d}\right\|}
$$

The boundary between two classes is a line (hyperplane) with equal distance between the centroid of the classes. After training of the documents, we compute $w$ and $d$. Thus, papers in pharmaceutical class are above the hyperplane $\left(\mathrm{w}^{*} \mathrm{~d}>\mathrm{b}\right)$ and papers in other class below hyperplane $\left(\mathrm{w}^{*} \mathrm{~d}<\mathrm{b}\right)$. we mean by $\mathrm{d}$

here is normalized weight for paper.

$$
\vec{w}=\vec{\mu}\left(c_{1}\right)-\vec{\mu}\left(c_{2}\right) \text { and } b=\hat{0} .5 *\left(\left|\vec{\mu}\left(c_{1}\right)\right|^{2}-\left|\vec{\mu}\left(c_{2}\right)\right|^{2}\right)
$$

For the testing phase, papers can be added as shown in figure 2. The labeling of the document is not required in this case. After the classification, the paper is added to pharmaceutical database if it is classified as pharmaceutical paper.

\subsection{The Okapi BM25 Ranking}

User can issue simple keyword like queries like "drug action" as shown in figure 5.

First, queries are preprocessed using preprocessing techniques as described in the methodology section. The relevant documents are retrieved and ranked from highest relevance score to lowest relevance score using the BM25 weighting scheme as shown in equation 6.

$$
\text { Score }_{\text {BM } 25}=\sum_{\mathrm{t} \in \mathrm{d} \cap \mathrm{q}} \frac{\ln (\mathrm{N}-\mathrm{df}+0.5)}{\mathrm{df}+0.5} \cdot \frac{\mathrm{tf}}{0.5+1.5 . \mathrm{dl} / \mathrm{avdl}+\mathrm{tf}}
$$

avdl: average length of all documents.

$\mathbf{N}$ is Number of documents in the collection. 


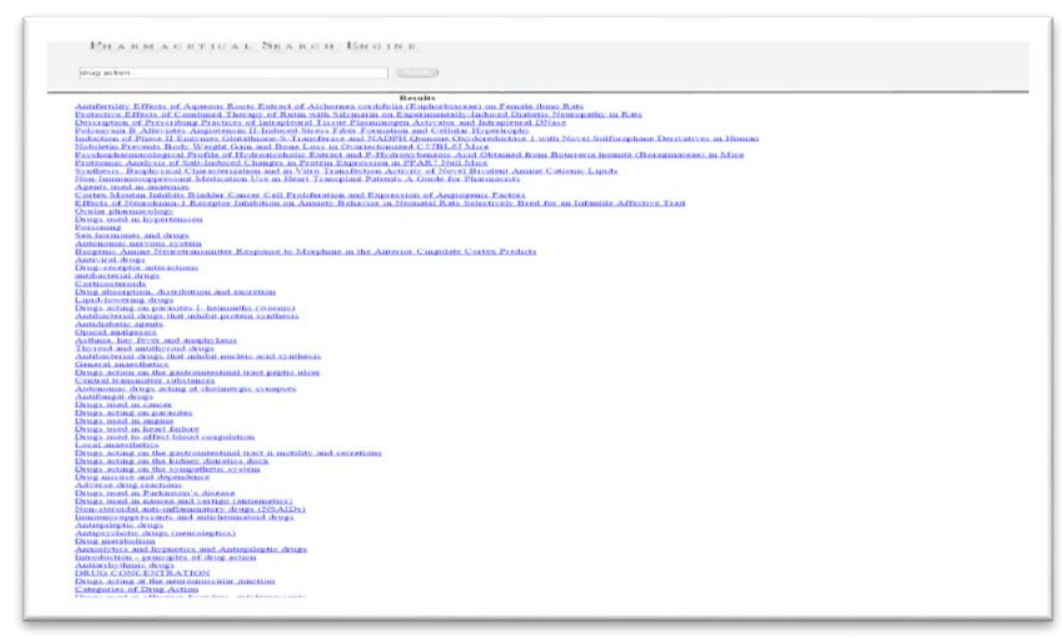

Figure 6. The retrieved result list

\section{EVALUATION}

For the supervised learning of the classifier, two different books for two classes are used.

First, Pharmaceutical book is converted to documents. Multiple chapters are included in one document depending upon the topic of the chapters. These documents are given to classifier for learning. For Pharmacology, medical Pharmacology book (Ritter et. al., 2008) is used. It is divided into 53 documents. The other class considered for classifier is computer science and operating system book (Silberschatz et. al.,2012)

is considered for the other class. Each chapter is divided into one document.

For the evaluation of classifier, abstract from the research papers are used. 86 paper from the two classes (16 related to computer science and 70 related to pharmacology \& medical pharmacology) are obtained from the internet (two examples papers are (Benbassat et al., 2014) (Egan et al., 2014)).

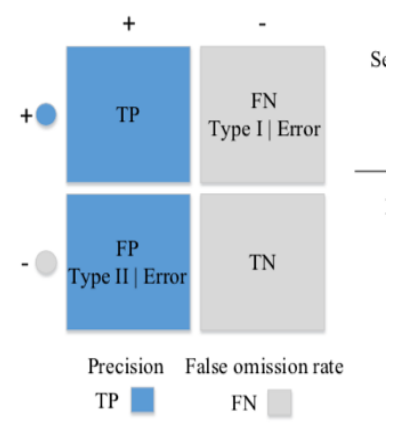

Figure 7. Confusion Matrix (from (Kowsari et. al, 2019))

The evaluation metric is based on a "confusion matrix" as shown in Figure 8 that comprises true positives (TP), false positives (FP), false negatives (FN), and true negatives (TN) (Kowsari et. al, 2019). The fraction of correct predictions over all predictions is called accuracy (Equation 7).

From 70 pharmacology \& medical pharmacology papers. Classifiers identify 63 papers as pharmacology with the accuracy of $90 \%$. One possible reason could be training data was only medical pharmacology and it didn't include general pharmacology data.

$$
\text { accuracy }=\frac{(T P+T N)}{(T P+F P+F N+T N)}
$$




\section{CONCLUSION}

There are a number of contributions of this work; (1) Document collection is built for the document categorization in the domain of Pharmacology (2) The development of document classifier using the Rocchio (3) Development of a Ranking module using BM25 weighting scheme (4) Document classifier is evaluated using 86 research papers and yields $90 \%$ results. The development of the interface for the administrator and user where administrator can easily classify new document and add it to the existing index for searching purposes. User can issue the query using the interface and obtain the ranked result list.

The PSE can be improved and extended in many ways such as improving the performance in terms of speed, error handling capabilities of the search engine. The system may be enhanced by using ontology of pharmaceutical science words and with the inclusion of crawler.

\section{REFERENCES}

Anon.,2019.thepharmeletter.[Online] Available at: https://www.thepharmaletter.com/article/60-of-online-search-resultsfor-pharmaceutical-drug-leads-to-fakes

Baeza-Yates R., Ribeiro-Neto, B., 2011. Modern Information Retrieval: The Concepts and Technology behind Search. s.l.:Addison-Wesley Professional.

Baeza-Yates, R., Calderón-Benavides, L. and González-Caro, C., 2006, October. The intention behind web queries. In International Symposium on String Processing and Information Retrieval (pp. 98-109). Springer, Berlin, Heidelberg.

Benbassat, B.V., Doneva, M. and Petrova, G.I., 2014. Pharmacotherapy Cost of Controlled Ovarian Hyperstimulation of in Vitro Fertilization-A Real Life Study. Pharmacology \& Pharmacy, 5(10), p.919.

Brin, S. and Page, L., 1998. The anatomy of a large-scale hypertextual web search engine. Computer networks and ISDN systems, 30(1-7), pp.107-117.

Chakrabarti, S., Punera, K. and Subramanyam, M., 2002, May. Accelerated focused crawling through online relevance feedback. In Proceedings of the 11th international conference on World Wide Web (pp. 148-159). ACM.

Craswell, N., Hawking, D. and Robertson, S., 2001, September. Effective site finding using link anchor information. In Proceedings of the 24th annual international ACM SIGIR conference on Research and development in information retrieval (pp. 250-257). ACM.

Croft, W. B., Metzler D.,Strohman T., 2009. Search Engines: Information Retrieval in Practices..:Pearson Education, Inc.

Davison, B.D., 2000, July. Topical locality in the web. In Proceedings of the 23rd annual international ACM SIGIR conference on Research and development in information retrieval (pp. 272-279). ACM.

Egan, G. and Pearson, G.J., 2014. Non-Immunosuppressant Medication Use in Heart Transplant Patients: A Guide for Pharmacists. Pharmacology \& Pharmacy, 5(10), p.950.

Fujii, A., 2008, April. Modeling anchor text and classifying queries to enhance web document retrieval. In Proceedings of the 17th international conference on World Wide Web (pp. 337-346). ACM

Goeuriot, L., Jones, G.J., Kelly, L., Müller, H. and Zobel, J., 2016. Medical information retrieval: introduction to the special issue. Information Retrieval Journal, 19(1-2), pp.1-5.

Han, E.H.S. and Karypis, G., 2000, September. Centroid-based document classification: Analysis and experimental results. In European conference on principles of data mining and knowledge discovery (pp. 424-431). Springer, Berlin, Heidelberg.

Harrell, F.E., 2015. Ordinal logistic regression. In Regression modeling strategies (pp. 311-325). Springer, Cham.

Jones, K.S., 1972. A statistical interpretation of term specificity and its application in retrieval. Journal of documentation, 28(1), pp.11-21.

Jones, K.S., 2004. IDF term weighting and IR research lessons. Journal of documentation, 60(5), pp. 521-523.

Kleinberg, J.M. 1999. Authoritative sources in a hyperlinked environment. J. ACM 46, 5 (September 1999), 604-632. DOI: https://doi.org/10.1145/324133.324140

Koopman, B., Zuccon, G., Bruza, P., Sitbon, L. and Lawley, M., 2016. Information retrieval as semantic inference: A Graph Inference model applied to medical search. Information Retrieval Journal, 19(1-2), pp.6-37.

Kowsari, K., Meimandi, K.J., Heidarysafa, M., Mendu, S., Barnes, L. and Brown, D., 2019. Text classification algorithms: A survey. Information, 10(4), p.150.

Larson, R., 2010. Introduction to information retrieval, Am. Soc. Inf. Sci. Technol. 
Lempel, R. and Moran, S., 2001. SALSA: the stochastic approach for link-structure analysis. ACM Transactions on Information Systems (TOIS), 19(2), pp. 131-160.

Li, L., Weinberg, C.R., Darden, T.A. and Pedersen, L.G., 2001. Gene selection for sample classification based on gene expression data: study of sensitivity to choice of parameters of the GA/KNN method. Bioinformatics, 17(12), pp. 1131-1142.

Lossio-Ventura, J.A., Jonquet, C., Roche, M. and Teisseire, M., 2016. Biomedical term extraction: overview and a new methodology. Information Retrieval Journal, 19(1-2), pp. 59-99.

Madhu, G., Govardhan, D.A. and Rajinikanth, D.T., 2011. Intelligent semantic web search engines: a brief survey. arXiv preprint arXiv:1102.0831.

Manevitz, L.M. and Yousef, M., 2001. One-class SVMs for document classification. Journal of machine Learning research, 2(Dec), pp. 139-154.

Manning, C. D., Schütze H., Raghavan P., 2008. Introduction to Information Retrieval. s.l.: Cambridge University Press New York, NY, USA .

Partalas, I., Kosmopoulos, A., Baskiotis, N., Artieres, T., Paliouras, G., Gaussier, E., Androutsopoulos, I., Amini, M.R. and Galinari, P., 2015. Lshtc: A benchmark for large-scale text classification. arXiv preprint arXiv:1503.08581.

Ritter, J., Lewis, L., Mant, T., Ferro, A., 2008. A Textbook of Clinical Pharmacology and Therapeutics. s.1.:CRC Press.

Rocchio, J., 1971. Relevance feedback in information retrieval. The Smart retrieval system-experiments in automatic document processing, pp. 313-323.

Silberschatz, A., Galvin, P.B., Gagne G., 2012. Operating System Concepts. s.1.:s.n.

Sowmya, B.J. and Srinivasa, K.G., 2016, October. Large scale multi-label text classification of a hierarchical dataset using Rocchio algorithm. In 2016 International Conference on Computation System and Information Technology for Sustainable Solutions (CSITSS) (pp. 291-296). IEEE

Tümer, D., Shah, M.A. and Bitirim, Y., 2009, May. An empirical evaluation on semantic search performance of keyword-based and semantic search engines: Google, yahoo, msn and hakia. In 2009 Fourth International Conference on Internet Monitoring and Protection (pp. 51-55). IEEE.

Xu, B.; Guo, X.; Ye, Y.; Cheng, J.,2012 An Improved Random Forest Classifier for Text Categorization. JCP 2012, 7 , 2913-2920. 